\title{
Discordance between perceived body size and actual body size and psychological wellbeing in adolescence: Evidence from the multi-ethnic DASH longitudinal study
}

\author{
C. Elia ${ }^{1}$, A. Karamanos ${ }^{2}$, A.M. João Silva ${ }^{3}$, M. O’Connor ${ }^{4}$, Y. Lu ${ }^{3}$, A. Dregan ${ }^{1}$ and S. Harding ${ }^{3}$ \\ ${ }^{1}$ Division of Primary Care and Public Health Research, King's College London, Guy's Campus, \\ London SE1 IUL, UK, \\ ${ }^{2}$ ESRC International Centre for Lifecourse Studies in Society and Health, Dept. Epidemiology and Health, \\ University College London, London WC1 6BT, \\ ${ }^{3}$ Cardiovascular Medicine \& Social Epidemiology group, Division of Diabetes \& Nutritional Sciences, \\ Kings College London, Waterloo campus, London SE1 9NH, UK and \\ ${ }^{4}$ Institute of Psychiatry, Psychology and Neuroscience, Kings College London, Denmark Hill, London SE5 9RJ, UK
}

Body image dissatisfaction is a source of distress for many young people and an important predictor of psychological function in young people. Weight misperceptions are common among adolescents in the United Kingdom ${ }^{(1)}$ and are correlated with depression, anxiety and low-self esteem ${ }^{(2)}$. The prevalence of obesity, body image dissatisfaction and the desire to be thin increase as children approach puberty, and can start as early as age nine ${ }^{(3)}$. The aim of this study was to evaluate whether the discordance between perceived body weight and measured Body Mass Index (BMI) influences psychological wellbeing among a multi-ethnic cohort of British adolescents.

Multivariate multilevel models were used to examine the association between psychological wellbeing, measured by the Total Difficulty Score (TDS) from Goodman's Strengths and difficulty Questionnaire ${ }^{(4)}$ (higher scores correspond with greater difficulties), and discordance among 3,228 adolescents in 49 London schools who participated in the Determinants of Adolescent Social wellbeing and Health longitudinal Study (DASH). Self-perception of body weight, measured anthropometry and psychosocial factors were collected at $11-13$ years and at 14-16 years follow-up.

Around $40 \%$ of adolescents had a discordant perception of weight (i.e. underestimated or overestimated actual weight), with girls being more likely than boys to be discordant $(\mathrm{p}<0.05)$. Discordance was related to age, ethnicity, gender, family life and racism. Among those who measured overweight/obese, more Black Caribbeans (11.9\%, $95 \%$ CI 9.7, 14.6) and Black Africans (10.5\%, $95 \%$ CI, 8.5, 12.8) than White British $(6.9 \%, 95 \%$ CI 5.40, 8.81) reported normal weight. Compared with concordance (accurate perception of weight), discordance was associated with higher TDS at $14-16 y(+0 \cdot 56,95 \%$ CI $0 \cdot 25,0 \cdot 87, p=0 \cdot 01)$, independent of all confounders. Increase in discordant perceptions between 11-13y and 14-16y was also associated with increasing TDS $(+0 \cdot 23,95 \%$ CI $0 \cdot 10,0 \cdot 46, p=0 \cdot 04)$, which attenuated on adjustment for racism. These effects did not vary by ethnicity.

These results confirm that misperception of weight is common and is associated with worse psychological wellbeing in adolescence. Interventions that engage with the underlying determinants of discordance, such as racism, and target families with young children could seem warranted.

1. Viner R, Haines M, Taylor S et al. (2006) Int J Obes 30, 1514-1521.

2. Goldfield GS, Moore C, Henderson K et al. (2010) J Sch Health 80, 186-192.

3. Micali N, De Stavola B, Ploubidis G et al. (2015) Br J Psychiatry 207, 320-327.

4. Goodman R (1997) J Child Psychol Psychiatry 38, 581-586. 